\title{
Manifestations buccales de la neutropénie congénitale sévère : à propos d'un cas
}

\author{
Lelièvre $\mathbf{J}^{\mathbf{1}}$, Boisramé-Gastrin $\mathrm{S}^{\mathbf{1}}$, Prédine-Hug $\mathrm{F}^{\mathbf{1}}$, Lemoine $\mathbf{P}^{\mathbf{2}}$, Pers $\mathrm{JO}^{\mathbf{1}}$ \\ ${ }^{1}$ UFR d'Odontologie, Service d'Odontologie, CHU, Brest, France \\ ${ }^{2}$ Service de Pédiatrie, CHU, Brest, France \\ lelievre.juju@voila.fr
}

Le syndrome de Kostmann ou neutropénie congénitale sévère constitue une forme rare de neutropénie profonde de l'enfant, caractérisée par un nombre de polynucléaires neutrophiles (PNN) inférieurs à 500 éléments par $\mathrm{mm}^{3}$, associé à un blocage de la maturation de la lignée granuleuse au stade promyélocyte/myélocyte, avec ou sans éosinophilie, avec ou sans monocytose (Kostmann 1956).

C'est un syndrome à transmission autosomique et la découverte de cette agranulocytose congénitale se fait dans la majorité des cas lors d'un syndrome infectieux néonatal. Le déficit en PNN favorise non seulement la survenue d'infections bactériennes et fongiques, mais il en modifie aussi l'expression et l'évolution : évolution vers la nécrose tissulaire, tendance à la diffusion tissulaire et à la cellulite. L'infection peut être localisée (cutanée, ORL, stomatologique, pneumologique) ou systémique ; elle peut alors constituer une urgence infectieuse majeure (Carlsson et al. 2006). Ainsi, avant l'apparition des facteurs de croissance hématopoïétiques, cette maladie était grevée d'une lourde morbidité et d'une mortalité précoce (Carlsson et Fasth 2001).

Le cas rapporté est celui d'un garçon de 12 ans qui présente une maladie parodontale agressive. Le diagnostic de syndrome de Kostmann a été posé à l'âge de 4 ans et des injections de G-CSF à la dose de $5 \mu \mathrm{g} . \mathrm{kg}^{-1}$ toutes les 48 heures ont été administrées. Malgré ce traitement, l'enfant a dû être hospitalisé à de nombreuses reprises pour des maladies infectieuses, le niveau des PNN restant très faible. Une autre thérapeutique a alors été initiée consistant en des injections sous-cutanées de $0,5 \mathrm{ml}$ de lénogastrim réalisées six jours sur sept à domicile. L'analyse génétique a permis de mettre en avant la mutation hétérozygote C194X du gène ELA-2 chez les enfants (une petite soeur est également atteinte) et chez le père. Ce dernier est asymptomatique. Il existerait une mosaïque germinale expliquant la survenue de la pathologie dans cette famille et l'absence de symptomatologie paternelle.

Sur le plan odontostomatologique, aucune prise en charge régulière n'avait été réalisée depuis le diagnostic du Kostmann jusqu'à l'âge de 12 ans. Devant le stade avancé de la parodontite agressive, les avulsions de 36 et 46 ont dû être réalisées sous antibioprophylaxie, et le traitement parodontal a permis de stabiliser la symptomatologie buccale.

Ce cas montre que les patients non allogreffés et ayant une neutropénie congénitale sévère, présentent une parodontite agressive malgré l'apport de facteurs de croissance. Par ailleurs, Carlsson (2006) a démontré que la mutation du gène ELA-2 constituait un facteur aggravant pour la prise en charge de cette maladie et de ses répercussions buccales. Une prise en charge odontologique rigoureuse est indispensable afin d'éviter une telle atteinte parodontale. 